\title{
On path integral for the radial Dirac equation
}

\author{
TAKASHI ICHINOSE*) \\ Department of Mathematics, Faculty of Science, Kanazawa University \\ Kanazawa, 920-1192, Japan
}

A path integral representation is given to the Green's function for the radial Dirac equation, by constructing a countably additive path space measure on the space of continuous paths living on the real half-line. An application is suggested to a problem in quantum field theory.

PACS: 03.65.Pm; 02.30.Sa

Key words: path integral, radial Dirac operator, Green's function

\section{Introduction and result}

The aim of this note is to make a survey of our mathematical rigorous result [12] on a path integral representation of the Green's function for the radial Dirac operator, and also to discuss a problem beyond that on the Yukawa 2 quantum field theory.

Consider the radial Dirac equation (e.g. [24, 25]) for a $\mathbf{C}^{2}$-valued function $u(r, t)={ }^{t}\left(u_{1}(r, t), u_{2}(r, t)\right)$

$$
\frac{\partial}{\partial t} u(r, t)=-\mathrm{i}\left[\tilde{\tau}_{k}+V(r)\right] u(r, t), \quad(r, t) \in \mathbf{R}^{+} \times \mathbf{R},
$$

with potential $V=V(r)$ being a real-valued function in $r \in \mathbf{R}^{+}=(0, \infty)$. The operator $\tilde{\tau}_{k}$ is the free radial Dirac operator with mass $m$, acting on the Hilbert space $L^{2}\left(\mathbf{R}^{+}, \mathrm{d} r\right) \otimes \mathbf{C}^{2}$ of the square-integrable $\mathbf{C}^{2}$-valued functions of $r>0$ with respect to the Lebesgue measure $\mathrm{d} r$ :

$$
\tilde{\tau}_{k}=-\mathrm{i} \sigma_{2} \frac{\partial}{\partial r}-\sigma_{1} \frac{k}{r}+m \sigma_{3}=\left(\begin{array}{cc}
0 & -1 \\
1 & 0
\end{array}\right) \frac{\partial}{\partial r}+\left(\begin{array}{cc}
m & -k / r \\
-k / r & -m
\end{array}\right)
$$

with the Pauli matrices $\sigma_{1}=\left(\begin{array}{cc}0 & 1 \\ 1 & 0\end{array}\right), \sigma_{2}=\left(\begin{array}{cc}0 & -\mathrm{i} \\ \mathrm{i} & 0\end{array}\right), \sigma_{3}=\left(\begin{array}{cc}1 & 0 \\ 0 & -1\end{array}\right)$. We are using the natural units $\hbar=c=1$ for Planck's constant $h$ divided by $2 \pi$ and the speed of light.

However, to simplify our argument, let us consider its unitary equivalent

$$
\tau_{k}=\mathrm{i} \sigma_{3} \frac{\partial}{\partial r}-\sigma_{1} \frac{k}{r}+m \sigma_{2}=\mathrm{i}\left(\begin{array}{cc}
1 & 0 \\
0 & -1
\end{array}\right) \frac{\partial}{\partial r}+\left(\begin{array}{cc}
0 & -k / r-\mathrm{i} m \\
-k / r+\mathrm{i} m & 0
\end{array}\right)
$$

Indeed there exists a unitary $2 \times 2$-matrix $N$ such that $N \sigma_{2} N^{-1}=-\sigma_{3}$ and $N \sigma_{1} N^{-1}=\sigma_{1}$.

\footnotetext{
*) E-mail: ichinose@kenroku.kanazawa-u.ac.jp
} 
The free radial Dirac operator arises from the spin-angular momentum decomposition of the free Dirac operator in 3-space dimensions. The nonzero integer $k$ $(= \pm 1, \pm 2, \ldots)$ represents an eigenvalue of the "spin-orbit operator".

In $[8,9,15]$, for the 1 -space-dimensional Dirac equation

$$
\frac{\partial}{\partial t} \psi(x, t)=-\left[\alpha \frac{\partial}{\partial x}+\mathrm{i} m \beta+V(x)\right] \psi(x, t), \quad(x, t) \in \mathbf{R} \times \mathbf{R},
$$

we have constructed a countably additive path space measure on the space of Lipschitz-continuous zigzag paths to give a path integral representation for its propagator. One of the decisive reasons why this is possible is that the fundamental solution $\exp \left[-\left(\alpha \partial_{x}+\mathrm{i} m \beta\right)\right](x, y)$ of the 1 -dimensional free equation is a ditribution of order zero in $(x, y) \in \mathbf{R} \times \mathbf{R}$, or in other words, the Cauchy problem is $L^{\infty}$ well-posed. The result has been refined in $[9,17,18]$ and finally in [10]. Some related works were also done in the meanwhile in $[2,4,5,20,26,6]$ and others.

Recently, in [13], we have, for the radial Dirac equation, explicitly computed the propagator, i.e. the integral kernel $\mathcal{K}_{t}(r, \rho)$ of $\mathrm{e}^{-\mathrm{i} t \tau_{k}}$ for $k=1$, but, showed that, though it turns out to be a distribution of order zero in $(r, \rho) \in \mathbf{R}^{+} \times \mathbf{R}^{+}$, there exists no countably additive path space measure to represent the propagator for the radial Dirac equation (1) because the free radial Dirac operator has a singularity at $r=0$ in its coefficients.

In this note we give a mathematical rigorous construction of a countably additive path space measure to represent, though not the propagator, the Green's function for the radial Dirac equation (1) with $\tau_{k}$ in (2) in place of $\tilde{\tau}_{k}$.

The idea is to combine our method of constructing a path space measure for the 1-dimensional Dirac equation in $[8,9,15,16,17,18]$, in particular, in [10] with the following simple/intriguing procedure of dealing with singularity to perform space-time transformation in path integrals used by physicists $([3,21,19]$, c.f. [7]):

$$
\frac{1}{A+B}=b \frac{1}{a(A+B) b} a=\int_{0}^{\infty} b \mathrm{e}^{-t[a(A+B) b]} a \mathrm{~d} t .
$$

Here we are expecting the $\mathrm{d} t$-integral on the right to vanish at infinity. The operator $\tau_{k}$ has a singularity at $r=0$ as in (2). However, multiply $\tau_{k}$ by some functions $a(r), b(r) \geq 0$ from the left and right sides, then $a(r) \tau_{k} b(r)$ becomes no more singular.

Let us take $a(r)=b(r)=r^{1 / 2}$. Then

$$
\begin{aligned}
r^{1 / 2} \tau_{k} r^{1 / 2} & =\mathrm{i} \sigma_{3} r^{1 / 2} \frac{\partial}{\partial r} r^{1 / 2}+\left(-k \sigma_{1}+m r \sigma_{2}\right)= \\
& =\mathrm{i}\left(\begin{array}{cc}
1 & 0 \\
0 & -1
\end{array}\right) r^{1 / 2} \frac{\partial}{\partial r} r^{1 / 2}+\left(\begin{array}{cc}
0 & -k-\mathrm{i} m r \\
-k+\mathrm{i} m r & 0
\end{array}\right) .
\end{aligned}
$$

For potentials $V(r)$, we assume that $V(r)=\frac{e}{r}+W(r)$ with a real constant $e$ satisfying $|e| \leq \sqrt{k^{2}-\frac{1}{4}}$, and $W(r)$ is $L_{\text {loc }}^{2}$ in $\mathbf{R}^{+}$being bounded near $r=0$. Note this class of real-valued functions $V(r)$ contains the Coulomb potential. 
We can show that the radial Dirac operator $\tau_{k}+V$ is essentially selfadjoint on $C_{0}^{\infty}\left(\mathbf{R}^{+}\right) \otimes \mathbf{C}^{2}$; its unique selfadjoint extension in $L^{2}\left(\mathbf{R}^{+}, \mathrm{d} r\right) \otimes \mathbf{C}^{2}$ has a real spectrum. It can be also shown that the relevant operator $r^{1 / 2}\left(\tau_{k}+V\right) r^{1 / 2}$ is essentially selfadjoint on $C_{0}^{\infty}\left(\mathbf{R}^{+}\right) \otimes \mathbf{C}^{2}$, which is a non-trivial result.

Since $r^{1 / 2} \tau_{k} r^{1 / 2}$ has no more singularity at $r=0$, so that $\mathrm{e}^{-\mathrm{i} t\left[r^{1 / 2}\left(\tau_{k}+V\right) r^{1 / 2}\right]}$ behaves better near $r=0$ than $\mathrm{e}^{-\mathrm{it}\left(\tau_{k}+V\right)}$, we can expect to construct a path space measure associated with the semigroup $\mathrm{e}^{-\mathrm{i} t\left[r^{1 / 2}\left(\tau_{k}+V\right) r^{1 / 2}\right]}$. Then the resolvent of $\tau_{k}+V$ is, by the Laplace transform of this semigroup like (3), formally expressed (with a suitable $\lambda \in \mathbf{C}$ ) as

$$
\begin{aligned}
\left(\tau_{k}+V-\lambda\right)^{-1}(r, \rho) & =r^{1 / 2}\left[\left(r^{1 / 2}\left(\tau_{k}+V-\lambda\right) r^{1 / 2}\right)^{-1}\right](r, \rho) \rho^{1 / 2}= \\
& =\mathrm{i} \int_{0}^{\infty} r^{1 / 2} \exp \left[-\mathrm{i} t\left(r^{1 / 2}\left(\tau_{k}+V-\lambda\right) r^{1 / 2}\right)\right] \rho^{1 / 2} \mathrm{~d} t
\end{aligned}
$$

This may give a path integral representation of the resolvent kernel/Green's function for the original radial Dirac operator $\tau_{k}+V(r)$, though we could not find such a representation for the propagator $\mathrm{e}^{-\mathrm{i} t\left(\tau_{k}+V\right)}$ itself.

We introduce the following notations. If $\mathbf{C}^{2}$ and $\left(\mathbf{C}^{2}\right)^{\prime}$ are, respectively, the space of the column 2-vectors and the space of the row 2-vectors, the space of the $2 \times 2$ complex matrices $M$ can be written as $M_{2}(\mathbf{C})=\mathbf{C}^{2} \otimes\left(\mathbf{C}^{2}\right)^{\prime}$. With $\overline{\mathbf{R}^{+}}=[0, \infty), C_{00}^{\infty}\left({\overline{\mathbf{R}^{+}}}^{2}\right) \otimes M_{2}(\mathbf{C})$ denotes the locally convex space of the $M_{2}(\mathbf{C})$ valued $C^{\infty}$ functions $M(r, \rho)$ in $\overline{\mathbf{R}^{+^{2}}}$, which have compact support and satisfy $\frac{\partial^{m+n}}{\partial r^{m} \partial \rho^{n}} M(r, \rho)=0$ at $r=0$ or $\rho=0$ for all $m, n \geq 0$. Let $C_{00}^{\infty}\left({\overline{\mathbf{R}^{+}}}^{2}\right)^{\prime} \otimes M_{2}(\mathbf{C})$ be its dual space. As $C_{0}^{\infty}\left(\mathbf{R}^{+2}\right) \otimes M_{2}(\mathbf{C})$ is a subspace of $C_{00}^{\infty}\left({\overline{\mathbf{R}^{+}}}^{2}\right) \otimes M_{2}(\mathbf{C})$, so $C_{00}^{\infty}\left(\overline{\mathbf{R}}^{2}\right)^{\prime} \otimes M_{2}(\mathbf{C})$ is a subspace of $\mathcal{D}^{\prime}\left(\mathbf{R}^{+2}\right) \otimes M_{2}(\mathbf{C})$ of the $M_{2}(\mathbf{C})$-valued distributions in $\mathbf{R}^{+^{2}}$. By $(\cdot, \cdot)$ and $\langle\cdot, \cdot\rangle$ we denote, respectively, the sesquilinear and bilinear inner products between a dual pairing.

We can show the following path integral representation for the resolvent $\left[\left(\tau_{k}+\right.\right.$ $\left.V \mp \mathrm{i} \varepsilon)^{-1}\right](r, \rho)$ for the radial Dirac operator $\tau_{k}+V(r), \varepsilon>0$. We denote by $|0, t|$ the interval $0 \leq s \leq t$ or $0 \geq s \geq t$ according as $t>0$ or $t<0$.

Theorem. Let $V(r)$ be a potential mentioned above. Then:

(i) there exists a $C_{00}^{\infty}\left({\overline{\mathbf{R}^{+}}}^{2}\right)^{\prime} \otimes M_{2}(\mathbf{C})(2 \times 2$-matrix distribution)-valued, countably additive path space measure $\mu_{t, 0}$ on the space $C\left(|0, t| \rightarrow \overline{\mathbf{R}^{+}}\right)$of the continuous paths $R:|0, t| \rightarrow \overline{\mathbf{R}^{+}}$such that the resolvent kernel $\left[\left(\tau_{k}+V \mp \mathrm{i} \varepsilon\right)^{-1}\right](r, \rho)$ for the radial Dirac operator admits a path integral representation: for every pair of $f, g \in C_{00}^{\infty}\left(\overline{\mathbf{R}^{+}}\right) \otimes \mathbf{C}^{2}$,

$$
\begin{gathered}
\left(f,\left(\tau_{k}+V \mp \mathrm{i} \varepsilon\right)^{-1} g\right)=\int_{0}^{\infty} \int_{0}^{\infty}{ }^{t} \overline{f(r)}\left[\left(\tau_{k}+V \mp \mathrm{i} \varepsilon\right)^{-1}\right](r, \rho) g(\rho) \mathrm{d} r \mathrm{~d} \rho= \\
=\mathrm{i} \int_{0}^{ \pm \infty} \mathrm{d} t \int_{C\left(|0, t| \rightarrow \overline{\mathbf{R}^{+}}\right)}\left\langle t \overline{f(R(t))}, \mathrm{d} \mu_{t, 0}(R) g(R(0))\right\rangle \times
\end{gathered}
$$




$$
\times R(t)^{1 / 2} R(0)^{1 / 2} \exp \left(-\int_{0}^{t}(\mathrm{i} V(R(s)) R(s) \pm \varepsilon R(s)) \mathrm{d} s\right) .
$$

In particular, the resolvent kernel $\left[\left(\tau_{k}+V \mp \mathrm{i} \varepsilon\right)^{-1}\right](r, \rho)$ is a distribution of order zero in $(r, \rho) \in \mathbf{R}^{+} \times \mathbf{R}^{+}$.

(ii) The measure $\mu_{t, 0}$ is concentrated on the set of continuous paths $R:|0, t| \rightarrow \overline{\mathbf{R}^{+}}$ for which there exists a finite partition:

$$
0=t_{0} \lesseqgtr t_{1} \lesseqgtr \ldots \lesseqgtr t_{n+1}=t
$$

of the interval $|0, t|$ such that for $t_{h-1} \lesseqgtr s \lesseqgtr t_{h}, 1 \leq h \leq n+1$,

$$
R(s)=R(0) \exp \left[ \pm\left(\sum_{p=1}^{h-1}(-1)^{p-1}\left(t_{p}-t_{p-1}\right)+(-1)^{h-1}\left(s-t_{h-1}\right)\right)\right]
$$

Thus each path $R(\cdot)$ is, for some finite $n$, an $n$-vertex piecewise smooth curve in the radial space-time.

(iii) Suppose that 0 is not an eigenvalue of the radial Dirac operator $\tau_{k}+V$. If the Green's function $G_{ \pm}(r, \rho)$ for $\tau_{k}+V$ exists, then it is a distribution of order zero in $(r, \rho) \in \mathbf{R}^{+^{2}}$, and given as follows : for every pair of $f, g \in C_{00}^{\infty}\left(\overline{\mathbf{R}^{+}}\right) \otimes \mathbf{C}^{2}$,

$$
\int_{0}^{\infty} \int_{0}^{\infty} t \overline{f(r)} G_{ \pm}(r, \rho) g(\rho) \mathrm{d} r \mathrm{~d} \rho=\lim _{\varepsilon \rightarrow+0} \text { the right-hand side of (5). }
$$

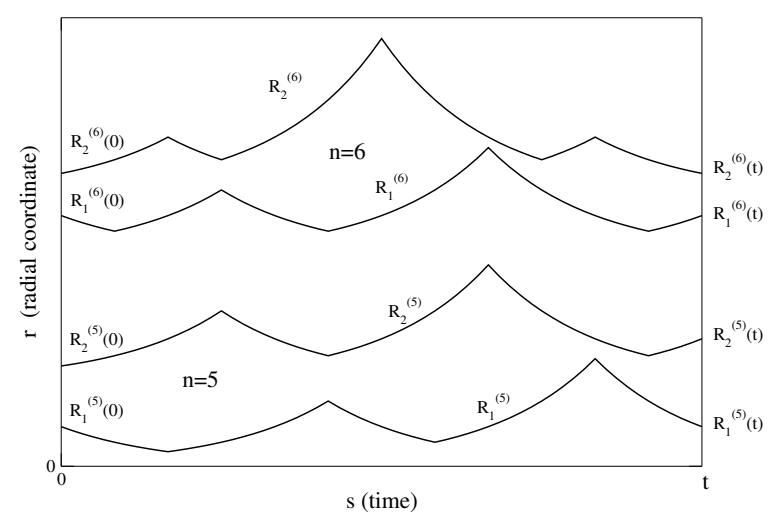

Fig. 1. Two 5, 6 times zigzag paths $R^{(5)}(\cdot), R^{(6)}(\cdot)$ are illustrated among the paths $R(\cdot)$ between the time interval $0 \leq s \leq t$ on which the path space measure $\mu_{t, 0}$ is concentrated.

The property of the support of the measure $\mu_{t, 0}$ in Theorem (ii) may be thought to describe a Zitterbewegung (see [23] or e.g. [24]) of a Dirac particle in radial spacetime, because the path $R(\cdot)$ in the support is a continuous zigzag curve which in every finite time interval is, for some finite $n$, on each of its $n$-partitioned short time 
intervals a segment exponentially growing or decreasing, though not a straight line segment. If formally expressed, equation (7) becomes, taking the "delta functions" at the 2 points $r>0$ and $\rho>0$ respectively for $f$ and $g$ :

$$
\begin{aligned}
G_{ \pm}(r, \rho)= & \mathrm{i} \lim _{\varepsilon \rightarrow+0} \int_{0}^{ \pm \infty} \mathrm{d} t \int_{C\left(|0, t| \rightarrow \overline{\mathbf{R}^{+}}\right), R(0)=\rho, R(t)=r} \mathrm{~d} \mu_{t, 0}(R) \times \\
& \times R(t)^{1 / 2} R(0)^{1 / 2} \exp \left(-\int_{0}^{t}(\mathrm{i} V(R(s)) R(s) \pm \varepsilon R(s)) \mathrm{d} s\right) .
\end{aligned}
$$

Section 2 gives an outline of how to construct the path space measure for the relevant operator $r^{1 / 2} \tau_{k} r^{1 / 2}$ to the radial Dirac operator $\tau_{k}$ in (2). In Section 3, we establish a path integral representation for the unitary group for the relevant operator to get to the Green's function of the original radial Dirac operator $\tau_{k}+$ $V(r)$. In Section 4, a problem is discussed on Yukawa 2 quantum field theory. The details of the contents until Section 3 is referred to [12].

\section{Outline of construction of the path space measure}

We consider only the case $t>0$, so that $|0, t|$ means the interval $0 \leq s \leq t$ or $[0, t]$. We want to construct our path space measure $\mu_{t, 0}$ on the space of the 1-dimensional continuous paths $R:[0, t] \rightarrow[0, \infty)=\overline{\mathbf{R}^{+}}$living on the closed halfline. The argument will go analogously with our argument used in [10] for the Dirac equation in 1-dimensional space $\mathbf{R}$.

Consider the Cauchy problem for the relevant operator $r^{1 / 2} \tau_{k} r^{1 / 2}$ in (4) instead of the original free radial Dirac operator $\tau_{k}$ in (2):

$$
\begin{aligned}
\partial_{t} u(r, t) & =-\mathrm{i}\left[r^{1 / 2} \tau_{k} r^{1 / 2}\right] u(r, t), & & r>0, t>0, \\
u(r, 0) & =g(r), & & t=0 .
\end{aligned}
$$

This is a hyperbolic system of the first order with the local propagation speed $c(r)=r$. Crucial is that this Cauchy problem is $L^{\infty}$ well-posed and satisfies:

Lemma 2.1. The solution $u(r, t)$ of (8) obeys for every $\mathbf{C}^{2}$-valued continuous function $g={ }^{t}\left(g_{1}, g_{2}\right)$ in $\overline{\mathbf{R}^{+}}$with compact support in a bounded interval $[0, c]$ with $c>0$ the inequality:

$$
\|u(\cdot, t)\|_{\infty}=\left\|\mathrm{e}^{-\mathrm{i} t\left[r^{1 / 2} \tau_{k} r^{1 / 2}\right]} g\right\|_{\infty} \leq \mathrm{e}^{|t|(M(c, k)+3 / 2)}\|g\|_{\infty},
$$

with $M(c, k)=m c+|k|$, and $u(\cdot, t)$ has support in the bounded closed interval

$$
\left[\mathrm{e}^{-|t|} \inf \operatorname{supp} g, \mathrm{e}^{|t|} \sup \operatorname{supp} g\right] \subset\left[0, \mathrm{e}^{|t|} c\right] .
$$

We begin to construct our path space measure $\mu_{t, 0}$.

Let $\dot{\mathbf{R}}^{+}=\overline{\mathbf{R}^{+}} \cup\{\infty\}=[0, \infty) \cup\{\infty\}$ be the one-point compactification of $\overline{\mathbf{R}^{+}}=[0, \infty)$, and, for each fixed $t>0$, let $\mathcal{R}_{t, 0}:=\prod_{[0, t]} \dot{\mathbf{R}}^{+}=\left(\dot{\mathbf{R}}^{+}\right)^{[0, t]}$ be the 
uncountably many copies of $\dot{\mathbf{R}}^{+}$. By the Tychonoff theorem $\mathcal{R}_{t, 0}$ is a compact Hausdorff space in the product topology. It may be regarded as the space of all paths $R:[0, t] \rightarrow \dot{\mathbf{R}}^{+}$, possibly discontinuous and possibly passing through zero and/or $\infty$.

Let $C\left(\mathcal{R}_{t, 0}\right)$ be the Banach space of the $\mathbf{C}$-valued continuous functions on $\mathcal{R}_{t, 0}$, and $C_{\text {fin }}\left(\mathcal{R}_{t, 0}\right)$ its subspace of those $\Psi$ in $C\left(\mathcal{R}_{t, 0}\right)$ for which there exist a finite partition of the interval $[0, t]$ :

$$
0=s_{0}<s_{1}<\cdots<s_{\ell-1}<s_{\ell}=t,
$$

and a $\mathbf{C}$-valued bounded continuous function $F\left(x_{0}, \ldots, x_{\ell}\right)$ on $\left(\dot{\mathbf{R}}^{+}\right)^{\ell+1}$ such that

$$
\Psi(R)=F\left(R\left(s_{0}\right), R\left(s_{1}\right), \ldots, R\left(s_{\ell}\right)\right) .
$$

In view of (4) put

$$
\begin{aligned}
C & :=-\mathrm{i}\left[r^{1 / 2} \tau_{k} r^{1 / 2}\right]=: A+B \\
A & :=-\mathrm{i}\left[\mathrm{i} r^{1 / 2} \sigma_{3} \frac{\partial}{\partial r} r^{1 / 2}\right]=\left(\begin{array}{cc}
1 & 0 \\
0 & -1
\end{array}\right) r^{1 / 2}\left(\frac{\partial}{\partial r}\right) r^{1 / 2}, \\
B & :=-\mathrm{i}\left(-k \sigma_{1}+m r \sigma_{2}\right)=\left(\begin{array}{cc}
0 & \mathrm{i} k+m r \\
\mathrm{i} k-m r & 0
\end{array}\right) .
\end{aligned}
$$

Let $K(t ; r, \rho)$ be the integral kernel of $\mathrm{e}^{t C}=\mathrm{e}^{-\mathrm{i} t\left[r^{1 / 2} \tau_{k} r^{1 / 2}\right]}$. Define, for each fixed $t>0$, a functional $L_{t, 0}(\Psi ; f, g)$ which is linear in $\Psi \in C_{\text {fin }}\left(\mathcal{R}_{t, 0}\right)$ and sesquilinear in $(f, g) \in\left[C_{00}^{\infty}\left(\overline{\mathbf{R}^{+}}\right) \otimes \mathbf{C}^{2}\right] \times\left[C_{00}^{\infty}\left(\overline{\mathbf{R}^{+}}\right) \otimes \mathbf{C}^{2}\right]$, by

$$
\begin{aligned}
L_{t, 0}(\Psi ; f, g)= & \int_{0}^{\infty} \mathrm{d} r_{\ell} \int_{0}^{\infty} \mathrm{d} r_{\ell-1} \cdots \int_{0}^{\infty} t \overline{f\left(r_{\ell}\right)} K\left(s_{\ell}-s_{\ell-1} ; r_{\ell}, r_{\ell-1}\right) \times \\
& \times K\left(s_{\ell-1}-s_{\ell-2} ; r_{\ell-1}, r_{\ell-2}\right) \cdot \ldots \cdot K\left(s_{1}-s_{0} ; r_{1}, r_{0}\right) \times \\
& \times F\left(r_{0}, r_{1}, \ldots, r_{\ell}\right) g\left(r_{0}\right) \mathrm{d} r_{0} .
\end{aligned}
$$

Then the right-hand side of (13) can be rewritten as

$$
\begin{aligned}
& L_{t, 0}(\Psi ; f, g)= \\
& =\int_{0}^{\infty} \int_{0}^{\infty}{ }^{t} \overline{f\left(r_{\ell}\right)} \mathrm{e}^{\Delta s_{\ell} C} \mathrm{e}^{\Delta s_{\ell-1} C} \ldots \mathrm{e}^{\Delta s_{1} C} F\left(r_{0}, r_{1}, \ldots, r_{\ell}\right) g\left(r_{0}\right) \mathrm{d} r_{\ell} \mathrm{d} r_{0}, \\
& \Delta s_{j}=s_{j}-s_{j-1}, \quad 1 \leq j \leq \ell,
\end{aligned}
$$

where $\mathrm{e}^{\Delta s_{j} C}$ is a linear operator (with integral kernel $K\left(s_{j}-s_{j-1} ; r_{j}, r_{j-1}\right)$ transforming the functions of $r_{j-1}$ to the functions of $r_{j}$.

We expand $\mathrm{e}^{t C} \equiv \mathrm{e}^{-\mathrm{i} t\left[r^{1 / 2} \tau_{k} r^{1 / 2}\right]}=\mathrm{e}^{t(A+B)}$ as a Dyson series

$$
\begin{aligned}
\mathrm{e}^{t C}=\mathrm{e}^{t A}+\sum_{n=1}^{\infty} & \int_{0}^{t} \mathrm{~d} t_{n} \int_{0}^{t_{n}} \mathrm{~d} t_{n-1} \cdots \int_{0}^{t_{2}} \mathrm{~d} t_{1} \mathrm{e}^{\left(t-t_{n}\right) A} \times \\
& \times B \mathrm{e}^{\left(t_{n}-t_{n-1}\right) A} B \cdots B \mathrm{e}^{\left(t_{2}-t_{1}\right) A} B \mathrm{e}^{t_{1} A} .
\end{aligned}
$$


Then, shifting all $B$ in the integrand of (16) to the left by use of the commutation relation

$$
\mathrm{e}^{t A} B=B(t) \mathrm{e}^{-t A}, \quad B(t):=\left[\mathrm{i} k \sigma_{1}-\mathrm{i} m r \sigma_{2}\left(\begin{array}{cc}
\mathrm{e}^{t} & 0 \\
0 & \mathrm{e}^{-t}
\end{array}\right)\right]
$$

we see there exists a $2 \times 2$-matrix $B_{n}\left(\Delta t_{2}, \ldots, \Delta t_{n+1}\right)$ dependent on $\Delta t_{j}:=t_{j}-t_{j-1}$, $1 \leq j \leq n+1$, and of course, dependent on $m, k, r$, such that

$$
\begin{aligned}
\mathrm{e}^{t C}= & \mathrm{e}^{t A}+\sum_{n=1}^{\infty} \int_{0}^{t} \mathrm{~d} t_{n} \int_{0}^{t_{n}} \mathrm{~d} t_{n-1} \cdots \int_{0}^{t_{2}} \mathrm{~d} t_{1} \times \\
& \times B_{n}\left(\Delta t_{2}, \ldots, \Delta t_{n+1}\right) \exp \left[\sum_{j=1}^{n+1}(-1)^{j-1} \Delta t_{j} A\right]= \\
= & : \sum_{n=0}^{\infty} C_{n}(t) .
\end{aligned}
$$

We can see that $C_{n}(t)$ satisfies the bound

$$
\left\|C_{n}(t) f\right\|_{\infty} \leq \frac{(m c+|k|)^{n}}{n !} t^{n} \mathrm{e}^{3 t / 2}\|f\|_{\infty},
$$

for all $f \in C_{0}^{\infty}\left(\mathbf{R}^{+}\right) \otimes \mathbf{C}^{2}$ with supp $f \subset[0, c]$. Moreover, since the integral kernel of

$$
\mathrm{e}^{t A}=\exp \left[-\mathrm{i} t\left(\mathrm{i} r^{1 / 2} \sigma_{3} \frac{\partial}{\partial r} r^{1 / 2}\right)\right]
$$

is given by

$$
\mathrm{e}^{t A}(r, \rho)=\left(\begin{array}{cc}
\mathrm{e}^{t / 2} \delta\left(\rho-r \mathrm{e}^{t}\right) & 0 \\
0 & \mathrm{e}^{-t / 2} \delta\left(\rho-r \mathrm{e}^{-t}\right)
\end{array}\right)
$$

the integral kernel of $C_{n}(t)$ has support

$$
\operatorname{supp} C_{n}(t)(r, \rho)=\left\{(\rho, r) \in \mathbf{R}^{+} \times \mathbf{R}^{+} ; \rho=r \exp \left( \pm\left[\sum_{j=1}^{n+1}(-1)^{j-1} \Delta t_{j}\right]\right)\right\} .
$$

Now substitute $\mathrm{e}^{t C}$ in (17) with $t$ replaced by $\Delta s_{j}$ into (15), then

$$
\begin{aligned}
L_{t, 0}(\Psi ; f, g)= & \sum_{n=0}^{\infty} \sum_{n_{1}+\cdots+n_{\ell}=n} \int_{0}^{\infty} \int_{0}^{\infty}{ }_{t} \overline{f\left(r_{\ell}\right)} \times \\
& \times C_{n_{\ell}}\left(\Delta s_{\ell}\right) \cdots C_{n_{2}}\left(\Delta s_{2}\right) C_{n_{1}}\left(\Delta s_{1}\right)\left[F\left(r_{0}, r_{1}, \ldots, r_{\ell}\right) g\left(r_{0}\right)\right] \mathrm{d} r_{\ell} \mathrm{d} r_{0}= \\
= & : \sum_{n=0}^{\infty} L_{t, 0}^{n}(\Psi ; f, g) .
\end{aligned}
$$

To construct $\mu_{t, 0}$ on $\mathcal{R}_{t, 0}$, the next lemma is crucial.

Lemma 2.2. (i) For each $t>0, L_{t, 0}(\Psi ; f, g)$ and $L_{t, 0}^{n}(\Psi ; f, g)$ are independent of the choice of $F$ corresponding to $\Psi \in C_{\mathrm{fin}}\left(\mathcal{R}_{t, 0}\right)$. 
(ii) If $f, g \in C_{0}^{\infty}\left(\mathbf{R}^{+}\right) \otimes \mathbf{C}^{2}$ with $\operatorname{supp} f$ or $\operatorname{supp} g \subseteq[0, c]$, then

$$
\begin{aligned}
& \left|L_{t, 0}(\Psi ; f, g)\right| \leq \mathrm{e}^{t(M(c, k)+3 / 2)}\|\Psi\|\|f\|_{1}\|g\|_{\infty}, \\
& \left|L_{t, 0}^{n}(\Psi ; f, g)\right| \leq \frac{M(c, k)^{n}}{n !} t^{n} \mathrm{e}^{3 t / 2}\|\Psi\|\|f\|_{1}\|g\|_{\infty},
\end{aligned}
$$

with $M(c, k):=m c+|k|$, for all $n=0,1,2, \ldots$

Proof. It is easy to see the assertion (i) is due to the semigroup property of

$$
\mathrm{e}^{t A}=\exp \left[-\mathrm{i} t\left(\mathrm{i} r^{1 / 2} \sigma_{3} \frac{\partial}{\partial r} r^{1 / 2}\right)\right] .
$$

(ii) (22) implies (21). So it suffices to show (22). This is seen as, by the bound (18) for $C_{n_{j}}\left(\Delta s_{j}\right)$ with $\Delta s_{j}$ instead of $t$,

$$
\begin{aligned}
\left|L_{t, 0}^{n}(\Psi ; f, g)\right| & \leq \sum_{n_{1}+\cdots+n_{\ell}=n} \prod_{p=1}^{\ell}\left[\frac{(m c+|k|)^{n_{p}}}{n_{p} !}\left(\Delta s_{p}\right)^{n_{p}} \mathrm{e}^{3 \Delta s_{p} / 2}\right]\|F\|_{\infty}\|f\|_{1}\|g\|_{\infty}= \\
& =\frac{M(c, k)^{n}}{n !}\left(\sum_{p=1}^{\ell} \Delta s_{p}\right)^{n} \exp \left(\frac{3}{2} \sum_{q=1}^{\ell} \Delta s_{q}\right)\|F\|_{\infty}\|f\|_{1}\|g\|_{\infty}= \\
& =\frac{M(c, k)^{n}}{n !} t^{n} \mathrm{e}^{3 t / 2}\|\Psi\|\|f\|_{1}\|g\|_{\infty} .
\end{aligned}
$$

Here we have used $\sum_{p=1}^{\ell} \Delta s_{p}=t$, and the identity

$$
\sum_{n_{1}+\ldots+n_{\ell}=n} \frac{\left(\Delta s_{1}\right)^{n_{1}}\left(\Delta s_{2}\right)^{n_{2}} \ldots\left(\Delta s_{\ell}\right)^{n_{\ell}}}{n_{1} ! n_{2} ! \ldots n_{\ell} !}=\frac{\left(\sum_{p=1}^{\ell} \Delta s_{p}\right)^{n}}{n !}=\frac{t^{n}}{n !} .
$$

This ends the proof of the lemma.

A consequence of this lemma is the following. As $C_{\text {fin }}\left(\mathcal{R}_{t, 0}\right)$ is dense in $C\left(\mathcal{R}_{t, 0}\right)$ by the Stone-Weierstrass theorem, so the inequalities (21) and (22) hold also for all $\Psi \in C\left(\mathcal{R}_{t, 0}\right)$. This means that for each pair $(f, g), L_{t, 0}(\cdot ; f, g)$ and $L_{t, 0}^{n}(\cdot ; f, g)$ are $\mathrm{C}$-valued continuous linear functionals on $C\left(\mathcal{R}_{t, 0}\right)$. So by the Riesz representation theorem, there exist $\mathbf{C}$-valued regular Borel measures $\mu_{t, 0 ; f, g}$ and $\mu_{t, 0 ; f, g}^{n}$ on $\mathcal{R}_{t, 0}$ for $n=0,1,2, \ldots$, such that for all $\Psi \in C\left(\mathcal{R}_{t, 0}\right)$,

$$
\begin{aligned}
& L_{t, 0}(\Psi ; f, g)=\int_{\mathcal{R}_{t, 0}} \mathrm{~d} \mu_{t, 0 ; f, g}(X) \Psi(X), \\
& L_{t, 0}^{n}(\Psi ; f, g)=\int_{\mathcal{R}_{t, 0}} \mathrm{~d} \mu_{t, 0 ; f, g}^{n}(X) \Psi(X) .
\end{aligned}
$$

Hence we can also see that there exist $C_{00}^{\infty}\left({\overline{\mathbf{R}^{+}}}^{2}\right)^{\prime} \otimes M_{2}(\mathbf{C})$ (distribution)-valued regular Borel measures $\mu_{t, 0}(\cdot)$ and $\mu_{t, 0}^{n}(\cdot)$ on $\mathcal{R}_{t, 0}$ such that for every pair $(f, g) \in$ $\left[C_{00}^{\infty}\left(\overline{\mathbf{R}^{+}}\right) \otimes \mathbf{C}^{2}\right] \times\left[C_{00}^{\infty}\left(\overline{\mathbf{R}^{+}}\right) \otimes \mathbf{C}^{2}\right]$,

$$
\left\langle{ }^{t} \bar{f}, \mu_{t, 0}(\cdot) g\right\rangle=\mu_{t, 0 ; f, g}(\cdot), \quad\left\langle{ }^{t} \bar{f}, \mu_{t, 0}^{n}(\cdot) g\right\rangle=\mu_{t, 0 ; f, g}^{n}(\cdot) .
$$


Here note it to hold that

$$
\begin{aligned}
C_{00}^{\infty}\left(\overline{\mathbf{R}}^{2}\right) \otimes M_{2}(\mathbf{C}) & =\left[C_{00}^{\infty}\left(\overline{\mathbf{R}^{+}}\right) \otimes \mathbf{C}^{2}\right] \hat{\otimes}\left[C_{00}^{\infty}\left(\overline{\mathbf{R}^{+}}\right) \otimes\left(\mathbf{C}^{2}\right)^{\prime}\right], \\
C_{00}^{\infty}\left(\overline{\mathbf{R}}^{+}\right)^{\prime} \otimes M_{2}(\mathbf{C}) & =\left[C_{00}^{\infty}\left(\overline{\mathbf{R}^{+}}\right)^{\prime} \otimes \mathbf{C}^{2}\right] \hat{\otimes}\left[C_{00}^{\infty}\left(\overline{\mathbf{R}^{+}}\right)^{\prime} \otimes\left(\mathbf{C}^{2}\right)^{\prime}\right],
\end{aligned}
$$

where these tensor products on the right are completed in the $\pi$ - or $\varepsilon$-tensor product topology, because $C_{00}^{\infty}\left(\overline{\mathbf{R}^{+}}\right)$and $C_{00}^{\infty}\left(\overline{\mathbf{R}^{+}}\right)^{\prime}$ are nuclear spaces.

It follows from the expansion (20) that

$$
\mu_{t, 0 ; f, g}(\cdot)=\sum_{n=0}^{\infty} \mu_{t, 0 ; f, g}^{n}(\cdot), \quad \mu_{t, 0}(\cdot)=\sum_{n=0}^{\infty} \mu_{t, 0}^{n}(\cdot),
$$

respectively, in the sense of expansion of the complex-valued measure and in the sense of expansion of the $2 \times 2$-matrix distribution-valued measure.

Finally we observe on which set of the paths the measures $\mu_{t, 0}$ and $\mu_{t, 0}^{n}$ are concentrated.

To do so, inspect the expression (17) in $C_{n_{j}}\left(\Delta s_{j}\right)$ :

$$
\begin{aligned}
C_{n_{j}}\left(\Delta s_{j}\right)= & \int_{s_{j-1}}^{s_{j}} \mathrm{~d} t_{j, n_{j}} \int_{s_{j-1}}^{t_{j, n_{j}}} \mathrm{~d} t_{j, n_{j}-1} \cdots \int_{0}^{t_{j, 2}} \mathrm{~d} t_{j, 1} \times \\
& \times B_{n_{j}}\left(\Delta t_{j, 2}, \ldots, l \Delta t_{j, n_{j}+1}\right) \exp \left(\sum_{q=1}^{n_{j}+1}(-1)^{q-1} \Delta t_{j, q} A\right),
\end{aligned}
$$

and consider their product

$$
\begin{aligned}
& C_{n_{\ell}}\left(\Delta s_{\ell}\right) \cdots C_{n_{2}}\left(\Delta s_{2}\right) C_{n_{1}}\left(\Delta s_{1}\right)= \\
& =\int \ldots \int \mathrm{d} t^{n_{\ell}} B_{n_{\ell}}(\cdots) \mathrm{e}^{(\cdots) A} \int \ldots \int \mathrm{d} t^{n_{\ell-1}} B_{n_{\ell-1}}(\cdots) \mathrm{e}^{(\cdots) A} \\
& \times \cdots \int \ldots \int \mathrm{d} t^{n_{2}} B_{n_{2}}(\cdots) \mathrm{e}^{(\cdots) A} \int \ldots \int \mathrm{d} t^{n_{1}} B_{n_{1}}(\cdots) \mathrm{e}^{(\cdots) A}
\end{aligned}
$$

to find its integral kernel. Here keep in mind the support property (19) of $C_{n}(t)$. We change the $n=n_{1}+\ldots+n_{\ell}$ integration variables $\left\{t_{j, q} ; 1 \leq q \leq n_{j}, 1 \leq j \leq \ell\right\}$ to $\left\{t_{1}, t_{2}, \ldots, t_{n}\right\}$ in the set of $\ell n_{j}$-pleces

$$
\begin{aligned}
\Delta\left(s_{1}, s_{2}, \ldots, s_{\ell}\right): 0= & s_{0} \leq t_{1} \leq t_{2} \leq \ldots \leq t_{n_{1}} \leq s_{1} \leq t_{n_{1}+1} \leq \\
& \leq \ldots \leq s_{\ell-1} \leq t_{n_{1}+\ldots+n_{\ell-1}+1} \leq \ldots \leq t_{n} \leq s_{\ell}=t .
\end{aligned}
$$

Then a little patient calculation shows that there exists a $2 \times 2$-matrix-valued function $B\left(t ; n_{1}, \ldots, n_{\ell}\right)$ such that

$$
\begin{aligned}
& C_{n_{\ell}}(\left.\Delta s_{\ell}\right) \ldots C_{n_{2}}\left(\Delta s_{2}\right) C_{n_{1}}\left(\Delta s_{1}\right)= \\
& \quad=\int \ldots \int_{\Delta\left(s_{1}, s_{2}, \ldots, s_{\ell}\right)} \mathrm{d} t_{n} \mathrm{~d} t_{n-1} \ldots \mathrm{d} t_{1} B\left(t ; n_{1}, \ldots, n_{\ell}\right) \exp \left(\sum_{h=1}^{n+1}\left(t_{h}-t_{h-1}\right) A\right) .
\end{aligned}
$$

Hence we can determine first the support of each measure $\mu_{t, 0}^{n}$ through the expression of $L_{t, 0}^{n}(\Psi ; f, g)$ in (20), and then the support of our $\mu_{t, 0}$, by (20) and (25), as the union of the supports of the measures $\mu_{t, 0}^{n}$. 


\section{Path integral representation}

We establish a path integral representation of Feynman-Kac type for the semigroup $\exp \left[-\mathrm{i} t\left(r^{1 / 2}\left(\tau_{k}+V(r)\right) r^{1 / 2}\right)\right]$ or the solution $u(r, t)$ to the Cauchy problem (8) for the relevant operator $r^{1 / 2}\left(\tau_{k}+V(r)\right) r^{1 / 2}=r^{1 / 2} \tau_{k} r^{1 / 2}+r V(r)$.

Theorem 3.1. For $\exp \left[-\mathrm{i} t\left(r^{1 / 2}\left(\tau_{k}+V(r)\right) r^{1 / 2}\right)\right]$ it holds that

$$
\begin{aligned}
& \left(f, \exp \left[-\mathrm{i} t\left(r^{1 / 2}\left(\tau_{k}+V(r)\right) r^{1 / 2}\right)\right] g\right)= \\
& \quad=\int_{C\left([0, t] \rightarrow \overline{\mathbf{R}^{+}}\right)}\left\langle{ }^{t} \overline{f(R(t))}, \mathrm{d} \mu_{t, 0}(R) g(R(0))\right\rangle \exp \left[-\mathrm{i} \int_{0}^{t} V(R(s)) R(s) \mathrm{d} s\right] .
\end{aligned}
$$

Proof. For $s>0$, define a linear operator $U(s)$ :

$$
\begin{aligned}
(U(s) g)(r) & =\left(\mathrm{e}^{-\mathrm{i} s\left[r^{1 / 2} \tau_{k} r^{1 / 2}\right]}\left(\mathrm{e}^{-\mathrm{i} s r V(r)}\right) g\right)(r)= \\
& =\int_{0}^{\infty} K(s ; r, \rho) \mathrm{e}^{-\mathrm{i} s V(\rho) \rho} g(\rho) \mathrm{d} \rho, \quad g \in C_{0}^{\infty}\left(\mathbf{R}^{+}\right) \otimes \mathbf{C}^{2} .
\end{aligned}
$$

The operator $U(s)$ is a contraction on $L^{2}\left(\mathbf{R}^{+}\right) \otimes \mathbf{C}^{2}$ because $\mathrm{e}^{-\mathrm{i}\left[r^{1 / 2} \tau_{k} r^{1 / 2}\right]}$ is unitary, and also bounded in the $L^{\infty}$ operator norm by Lemma 2.1. Then for every integer $n>0$ with $s_{h-1}:=\frac{(h-1) t}{n}, h=1,2, \ldots, n+1$, we find by (13) that for $f \in$ $C_{0}^{\infty}\left(\mathbf{R}^{+}\right) \otimes \mathbf{C}^{2}$,

$$
\begin{aligned}
&\left(f, U(t / n)^{n} g\right)= \overbrace{\int_{0}^{\infty} \cdots \int_{0}^{\infty}}^{(n+1)-\text { times }} t \overline{f\left(r_{n}\right)} \overleftarrow{\prod}_{h=1}^{n} K\left(\frac{t}{n} ; r_{h}-r_{h-1}\right) \times \\
& \quad \times \exp \left[-\mathrm{i} \sum_{h=1}^{n} V\left(r_{h-1}\right) r_{h-1}\left(\frac{t}{n}\right)\right] g\left(r_{0}\right) \mathrm{d} r_{0} \mathrm{~d} r_{1} \ldots \mathrm{d} r_{n}= \\
&=\int_{C\left([0, t] \rightarrow \overline{\left.\mathbf{R}^{+}\right)}\right.}\left\langle t \overline{f(R(t))}, \mathrm{d} \mu_{t, 0}(R) g(R(0))\right\rangle \times \\
& \quad \times \exp \left[-\mathrm{i} \sum_{h=1}^{n} V\left(R\left(s_{h-1}\right)\right) R\left(s_{h-1}\right) \frac{t}{n}\right] .
\end{aligned}
$$

Then, as $n \rightarrow \infty$, the first member above converges, by the Trotter product formula (e.g. [RS]), to $\left(f, \exp \left[-\mathrm{i} t\left(r^{1 / 2}\left(\tau_{k}+V(r)\right) r^{1 / 2}\right)\right] g\right)$, since $r^{1 / 2}\left(\tau_{k}+V(r)\right) r^{1 / 2}=$ $r^{1 / 2} \tau_{k} r^{1 / 2}+r V(r)$ is essentially selfadjoint on $C_{0}^{\infty}\left(\mathbf{R}^{+}\right) \otimes \mathbf{C}^{2}$.

On the other hand, the last member converges, by the Lebesgue convergence theorem, to

$$
\int_{C\left([0, t] \rightarrow \overline{\mathbf{R}^{+}}\right)}\left\langle{ }^{t} \overline{f(R(t))}, \mathrm{d} \mu_{t, 0}(R) g(R(0))\right\rangle \exp \left[-\mathrm{i} \int_{0}^{t} V(R(s)) R(s) \mathrm{d} s\right] .
$$

Thus we have shown Theorem 3.1. 
Remark. The Trotter product formula used here is the unitary one, which usually converges only in the strong operator topology. Though it is irrelevant to the above proof, we would like to note here that there are some nontrivial special cases where the unitary Trotter product formula converges even in the operator norm, including the case for the Dirac operator $-\mathrm{i} \sum_{j=1}^{3} \alpha_{j} \partial_{x_{j}}+m \beta+V(x)$ with suitable potentials $V(x)$. This fact has recently been discussed in [14] (see also [11]).

Now, the proof of the main theorem is immediate. In Theorem 3.1, with $\varepsilon>0$ consider $\exp \left[-\mathrm{i} t\left(r^{1 / 2}\left(\tau_{k}+V(r)\right) r^{1 / 2} \mp \mathrm{i} \varepsilon r\right)\right]$ instead of $\exp \left[-\mathrm{i} t\left(r^{1 / 2}\left(\tau_{k}+V(r)\right) r^{1 / 2}\right)\right]$. Then we can get by Theorem 3.1 the expression (26) corresponding to this case. Then taking into account the relation between the radial Dirac operator $\tau_{k}+V(r)$ and the relevant operator $r^{1 / 2}\left(\tau_{k}+V(r)\right) r^{1 / 2}$ with the relation (3) in mind, we have only take the Laplace transform of both sides to get (5). This shows the main theorem.

\section{Application to a problem in Yukawa 2 quantum field theory}

Battle and Rosen [1] used Vekua-Bers's theory of generalized analytic functions to show the FKG inequality for Yukawa 2 quantum field theory. The $Y_{2}$ measure is formally given by

$$
\begin{aligned}
\mathrm{d} \nu(\phi) & :=\frac{1}{Z} \mathrm{e}^{W(\phi)} \prod_{x \in \mathbf{R}^{2}} \mathrm{~d} \phi(x), \\
W(x) & :=-\frac{1}{2}\left(\phi,\left(-\Delta+m_{b}^{2}\right) \phi\right)+\operatorname{Tr} K-\frac{1}{2}: \operatorname{Tr} K^{*} K:+\operatorname{Tr} \ln (1-K),
\end{aligned}
$$

with a normalization constant $Z$, where $\phi$ is the Boson field with Boson mass $m_{b}>0 . K$ is an operator with integral kernel

$$
K(x, y):=S(x, y) \phi(y) \chi_{\Lambda}(y),
$$

with $\chi_{\Lambda}$ the indicator function of the set $\Lambda \subset \mathbf{R}^{2}$, and for $x=\left(x_{0}, x_{1}\right) \in \mathbf{R}^{2}$,

$$
\begin{aligned}
S(x, y) & :=\left(-\beta \partial_{x}+m_{f}\right)^{-1} \Gamma, \quad \beta \partial_{x}=\beta_{0} \partial_{0}+\beta_{1} \partial_{1}, \\
\beta_{0} & :=\left(\begin{array}{ll}
0 & 1 \\
1 & 0
\end{array}\right)=\sigma_{1}, \quad \beta_{1}:=\left(\begin{array}{cc}
1 & 0 \\
0 & -1
\end{array}\right)=\sigma_{3},
\end{aligned}
$$

with $m_{f} \geq 0$ being the Fermi mass. They considered the two cases
a) $\Gamma:=\left(\begin{array}{ll}1 & 0 \\ 0 & 1\end{array}\right)=I_{2} \quad\left(\right.$ scalar $\left.Y_{2}\right)$
b) $\Gamma:=\left(\begin{array}{cc}0 & -1 \\ 1 & 0\end{array}\right)=-\mathrm{i} \sigma_{2} \quad\left(\right.$ pseudo-scalar $\left.\mathrm{Y}_{2}\right)$.

Then the FKG Inequality (like $\langle f g\rangle \geq\langle f\rangle\langle g\rangle$ ) holds if $\frac{\delta^{2} W}{\delta \phi(x) \delta \phi(y)} \geq 0, x \neq y$. This condition is equivalent to showing that $\operatorname{tr} S^{\prime}(x, y) S^{\prime}(y, x) \leq 0, x \neq y$, where tr 
strands for the $2 \times 2$ - matrix trace, and $S^{\prime}:=(1-K)^{-1} S$ is the Green's function (vanishing at $\infty$ ) for the 2-dimensional Euclidean Dirac equation

$$
\left[\Gamma^{-1}\left(-\beta \partial_{x}+m_{f}\right)-\phi(x) \chi_{\Lambda}(x)\right] S^{\prime}(x, y)=\delta(x-y) .
$$

The approach of the present note will give a path integral representation for this Green's function $S^{\prime}(x, y)$, though it may be the case where the function $\phi$ and the set $\Lambda$ are rotation-invariant on $\mathbf{R}^{2}$, and be suggested to give an alternative proof to the FKG inequality. The issue will be discussed elsewhere.

The author wishes to thank the organizers of this Path Integral Conference, Prague, June 2005, for their invitation. The research was partially supported by the Grant-in-Aid for Scientific Research (B) No. 16340038 and Grand-in-Aid for Exploratory Research No. 17654030, Japan Society for the Promotion of Science.

\section{References}

[1] G.A. Battle and L. Rosen: J. Statist. Phys. 22 (1980) 123.

[2] Ph. Blanchard, Ph. Combe, M. Sirugue and M. Sirugue-Collin: Probabilistic solution of the Dirac Equation, Path integral representation for the solution of the Dirac equation in presence of an electromagnetic field. Bielefeld, BiBoS Preprints Nos. 44, 66, 1985;

Ph. Combe, M. Sirugue and M. Sirugue-Collin: in: Proceedings of the VIIIth International Congress on Mathematical Physics $(M \cap \Phi)$, (M. Mebkhout and R. Sénéor, eds.), Marseille, 1986, World Scientific, Singapore, 1987, pp. 421-430.

[3] H. Duru and H. Kleinert: Phys. Lett. B 84 (1979) 185;

H. Duru and H. Kleinert: Fortschr.Phys. 30 (1982) 401.

[4] B. Gaveau: J. Functional Analysis 58 (1984) 310.

[5] B. Gaveau, T. Jacobson, M. Kac and L.S. Schulman: Phys. Rev. Lett. 53 (1984) 419.

[6] B. Gaveau and L.S. Schulman: Phys. Rev. D 36 (1987) 1135;

B. Gaveau and L.S. Schulman: Nuovo Cimento D 11 (1989) 31.

[7] Ch. Grosche and F. Steiner: Handbook of Feynman Path Integrals. Springer Tracts in Modern Physics, 145, Springer, Berlin Heidelberg, 1998.

[8] T. Ichinose: Proc. Japan Acad. A 58 (1982) 290;

T. Ichinose: Physica A 124 (1984) 419.

[9] T. Ichinose: Duke Math. J. 51 (1984) 1.

[10] T. Ichinose: Sugaku Expositions 6 (1993) 13, American Mathematical Society.

[11] T. Ichinose: in: A Garden of Quanta, Essays in Honor of Hiroshi Ezawa, World, Singapore, Singapore, 2003, pp. 77-93.

[12] T. Ichinose: J. Math. Phys. 46 (2005) 022103.

[13] T. Ichinose and B. Jefferies: J. Math. Phys. 43 (2002) 3963.

[14] T. Ichinose and Hideo Tamura: Lett. Math. Phys. 71 (2004) 65.

[15] T. Ichinose and Hiroshi Tamura: J. Math. Phys. 25 (1984) 1810. 
[16] T. Ichinose and Hiroshi Tamura: in: Proceedings of a Workshop "Functional Integration with Emphasis on the Feynman Integral", Sherbrooke, Quebec, Canada, July 21-32, 1986; Supplemento ai Rendiconti del Circolo Matematico di Palermo, Serie II, numero 17 (1987), pp. 237-248.

[17] T. Ichinose and Hiroshi Tamura: J. Math. Phys. 29 (1988) 103.

[18] T. Ichinose and Hiroshi Tamura: Progr. Theor. Phys. Suppl. 92 (1987) 144.

[19] A. Inomata, H. Katsuraji and C.C. Gerry: Path Integrals and Coherent States of $\mathrm{SU}(2)$ and $\mathrm{SU}(1,1)$, World Scientific, Singapore, 1992.

[20] T. Jacobson: J. Phys. A 17 (1984) 2433.

[21] H. Kleinert: Path Integrals in Quantum Mechanics Statistics and Polymer Physics, 2nd edition, World Scientific, Singapore, 1995.

[22] M. Reed and B. Simon: Methods of Modern Mathematical Physics, I. Functional Analysis, Revised and Enlarged Edition, Academic Press, New York, 1980.

[23] E. Schrödinger: Über die kräftefreie Bewegung in der relativistischen Quantenmechanik, Sitzungsber. Press. Akad. Wiss. Berlin, Phys.-math. Kl. 24 (1930), 418428.

[24] B. Thaller: The Dirac Equation. Texts and Monographs in Physics, Springer Verlag, Berlin-Heidelberg-New York, 1992.

[25] J. Weidmann: Spectral Theory of Ordinary Differential Operators. Lect. Notes in Math. 1258, Springer, Berlin, 1987.

[26] T. Zastawniak: Bull. Polish Acad. Sci. Math. 36 (1988) 341. 